\title{
Synthesis, crystal structure and spectroscopic properties of a novel tricyclic cinnoline derivative
}

\author{
Yuehua Yuan ${ }^{\mathrm{a}}$, Maozhong Tian ${ }^{\mathrm{a}, *}$, Qiangfeng Yin ${ }^{\mathrm{b}}$, Feng Feng ${ }^{\mathrm{a}, *}$ \\ ${ }^{a}$ College of Chemistry and Environmental Engineering, Shanxi Datong University, Datong \\ 037009, P. R. China. \\ ${ }^{b}$ Product Engineering Department, Chemical Engineering Institute, \\ East China University of Science and Technology, Shanghai 200237, P. R. China. \\ * Corresponding author. Tel.: +086-352-7624721; Fax: +086-352-7624721 \\ E-mail address:_tmzhong2002@163.com; feng-feng64@263.net
}

\begin{abstract}
A facile, efficient synthesis of the novel cinnoline derivative (E)-2,2'-(3-((3-bromophenyl) imino)-5-(tert-butyl)-3H-pyrrolo[3,2,1-ij]cinnoline-2,8-diyl)bis(propan-an-2-ol) (1) is reported. Richter cyclization of an o-ethynenearyldiazonium salt gave the cinnoline derivative which underwent a cyclization reaction to form the unexpected tricyclic compound $\mathbf{1}$. The absorption and fluorescence properties of cinnoline $\mathbf{1}$ were investigated in various solvents. A single crystal of $\mathbf{1}$ was grown and analyzed by X-ray diffraction analysis. The results showed that the inclusion compound was formed between the host (cinnoline 1) and the guest (acetone) in the crystal.
\end{abstract}

Keywords: Cinnoline derivative; Synthesis; Absorption spectra; Fluorescence quantum yields; Single crystal; Hydrogen bond

\section{Introduction}

Cinnoline derivatives are an important class of heterocyclic compounds showing biological activities [1]. In recent years, a large number of cinnoline compounds were 
found to exhibit interesting pharmacological properties including anticancer, anti-inflammatory and antibacterial [2]. Several methods for the synthesis of cinnolines have now been reported [3-5]. The cyclization of ortho-alkynylbenzenediazonium salts was discovered by Richter [6]. The chemistry of ortho-ethynyl-substituted arenediazonium salts has attracted considerable attention in recent yesrs [7-8]. These substances have proven to be valuable precursors of cinnoline derivatives enabling various types of substituents in the aromatic ring accessible.

In this paper an unexpected tricyclic cinnoline 1 was reported, which was synthesized via the cyclization reaction of an ortho-ethynyl-substituted cinnoline derivative under rather mild reaction condition.

\section{Experimental}

\subsection{Materials}

All reactions were carried out under a nitrogen atmosphere. All the materials were obtained from commercial suppliers and were used without further purification.

\subsection{Instruments}

${ }^{1} \mathrm{H}-\mathrm{NMR}$ and ${ }^{13} \mathrm{C}-\mathrm{NMR}$ spectra were recorded at $400 \mathrm{MHz}$ and $100 \mathrm{MHz}$, respectively. Chemical shifts $(\delta)$ were expressed in ppm relative to TMS and coupling constants $(J)$ are in Hz. Matrix-assisted laser desorption/ionization reflectron time-of-flight (MALDI-TOF) mass spectrometry was performed with a BrukerBiflex III mass spectrometer. UV-vis absorption spectra were measured on a PerkinElmer Lambda 35 spectrophotometer in a quartz cell. Fluorescence excitation and emission spectra were recorded by using a Hitachi F 2500 Fluorescence Spectrophotometer in a quartz cell. The crystal data collection of compound $\mathbf{1}$ was performed on 
a CrysAlisPro diffractometer.

\section{Results and discussion}

\subsection{Synthesis of cinnoline 1}

The synthetic strategy to the new cinnoline $\mathbf{1}$ is depicted in Scheme 1 . The reaction between compound $\mathbf{2}$ and 3-bromoaniline offered cinnoline $\mathbf{1}$ as an orange solid (51.7\%) after purification by column chromatography. Compound $\mathbf{1}$ was synthesized from the ortho-ethenyl substituted cinnoline derivative 2 in good yield (78\%) upon refluxing in THF.

\subsection{Spectroscopic properties}

The new cinnoline derivative $\mathbf{1}$ was dissolved in different solvents to enable its spectroscopic properties to be studied (Table S1). The absorption spectra of the cinnoline 1 are similar in shape in these solvents, showing a prominent, broad absorption feature around $388 \mathrm{~nm}$ (Fig S1). The excitation and emission maxima of cinnoline 1 have no obvious shift in organic solvents with various polarities (Fig S2). Cinnoline 1 has a maximum of excitation (400-417 nm) and emission (452-455 nm) within a narrow wavelength range in the five solvents.

As seen from the data in Table S1, the fluorescence quantum yields $\left(\Phi_{f}\right)$ of cinnoline $\mathbf{1}$ in different solvents are low which may arise as a result of the possible photoinduced electron transfer from the amino to the tricyclic cinnoline and internal heavy atom effect (bromine atom).

The molar absorption coefficients for cinnoline $\mathbf{1}$ are high and lie within the range of $1.08 \times$ $10^{4}-2.38 \times 10^{4} \mathrm{~L} \cdot \mathrm{mol}^{-1} \cdot \mathrm{cm}^{-1}$ in the five solvents. The fact that the molar absorption coefficients are higher than $10^{4} \mathrm{~L} \cdot \mathrm{mol}^{-1} \cdot \mathrm{cm}^{-1}$ indicate that the long-wavelength band of the absorption spectra is a band of charge transfer which occurs as a result from a $\pi \rightarrow \pi^{*}$ electron transfer during the $S_{0} \rightarrow S_{1}$ 
transition.

\subsection{Single crystal $X$-ray structural analysis of cinnoline $\mathbf{1}$}

The ORTEP plot of the molecular structure, with the atom labelling, of cinnoline $\mathbf{1}$ is showed in Fig. 1. Crystal data and structure refinement for cinnoline 1 were shown in Table S2. The relevant bond lengths and angles are listed in Table S3.

An examination of the deviations from the least-squares planes through the individual rings showed that the rings $\mathrm{A}(\mathrm{C} 1-\mathrm{C} 6), \mathrm{B}(\mathrm{C} 1, \mathrm{C} 6, \mathrm{C} 9, \mathrm{C} 10, \mathrm{~N} 1, \mathrm{~N} 2)$, and $\mathrm{C}(\mathrm{C} 5-\mathrm{C} 8, \mathrm{~N} 1)$ are almost planar. The cinnoline ring systems were planar, with dihedral angle of $4.86(0)^{\circ}$ between the planes of ring $\mathrm{A}$ and ring $\mathrm{B}$. The cinnoline ring systems (the ring $\mathrm{A}$ and ring $\mathrm{B}$ ) and the ring $\mathrm{C}$ are almost coplanar [r.m.s. deviation $=0.043 \mathrm{~nm}]$. The plane of the phenyl ring $(\mathrm{C} 22-\mathrm{C} 25)$ are twisted out of the plane of central tricyclic cinnoline unit on the one side. The dihedral angle between the plane of phenyl ring (C22-C27) and that of ring $\mathrm{A}$ is 71.2(1). Obviously, the bond length of $\mathrm{C} 7-\mathrm{C} 8$ $(1.356(3) \AA$ A ) is in good agreement with a carbon-carbon double bond. The bond length of C9-N2 (1.301(3) $\AA$ ) is in good agreement with a carbon-nitrogen double bond. Moreover, the bond

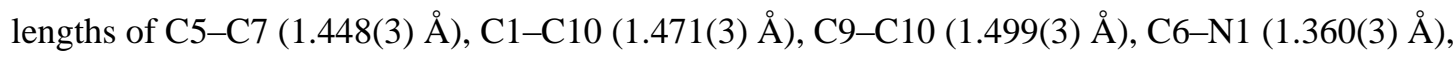
and C8-N1 (1.416(2) ̊) are, as expected, shorter than a normal carbon-carbon single bond and carbon-nitrogen single bond due to a conjugation effect. Therefore, it could be suggested that the ring $\mathrm{A}$ and ring $\mathrm{B}$ is highly conjugated with the $\mathrm{C}$ ring.

The molecules are linked into a zigzag one-dimensional chain along the a-axis by intermolecular $\mathrm{O}-\mathrm{H} \cdots \mathrm{O}\left(\mathrm{O}(1)-\mathrm{H}(1) \cdots \mathrm{O}(2)^{\mathrm{i}}: \mathrm{O}-\mathrm{H}=0.084 \mathrm{~nm}, \mathrm{H} \cdots \mathrm{O}=0.198 \mathrm{~nm}, \mathrm{O} 1 \cdots \mathrm{O} 4=\right.$ $0.2822(2) \mathrm{nm}, \mathrm{O}(1)-\mathrm{H}(1) \cdots \mathrm{O}(2)=175^{\circ}$, symmetry code: (i) $\mathrm{x}+1, \mathrm{y}, \mathrm{z}$ ) hydrogen bonds (Fig S3). The oxygen atom plays a significant role in the crystal packing as $\mathrm{O}(2)$ is the acceptor and $\mathrm{O}(1)$ is 
a donor in one hydrogen bond. The crystal-packing diagram shows that the introduction of two crystallizing acetone molecules in the complex successfully leads to the self assembly of the molecules by intermolecular hydrogen bonds.

\section{Conclusion}

In conclusion, we have presented the synthesis of a novel and unexpected tricyclic cinnoline $\mathbf{1}$. The introduction of two crystallizing acetone molecules in the complex successfully leads to the self assembly of these monomeric units by intermolecular hydrogen bonds. The fluorescence quantum yields of cinnoline $\mathbf{1}$ are low in various solvents due to the photoinduced electron transfer and internal heavy atom effect (bromine atom).

\section{Acknowledgments}

We thank the National Science Foundation of China (project 21175085, 21375083 and 21106044) and the Shanxi Scholarship Council of China (No. 200310) for support of this work.

\section{References}

[1] Li S, Zhao YF, Wang KW, Gao YL, Han JM, Cui BB, Gong P. Discovery of novel 4-(2-fluorophenoxy)quinoline derivatives bearing 4-oxo-1,4-dihydrocinnoline-3-carboxamide moiety as c-Met kinase inhibitors. Bioorg Med Chem 2013; 21 (11): 2843-55.

[2] Khalafy J, Rimaz M, Ezzati M, Prager RH. A Green One-Pot Protocol for Regioselective Synthesis of New Substituted 7,8-Dihydrocinnoline-5(6H)-ones. Bull Korean Chem Soc 2012; 33 (9): 2890-6.

[3] Vinogradova OV, Balova IA, Popik VV. Synthesis and reactivity of cinnoline-fused cyclic enediyne. J Org Chem 2011; 76 (16): 6937-41.

[4] Dey R, Ranu BC. A convenient and efficient protocol for the synthesis of 4(1H)-cinnolones, 
1,4-dihydrocinnolines, and cinnolines in aqueous medium: application for detection of nitrite ions. Tetrahedron 2011; 67 (46): 8918-24.

[5] Muralirajan K, Cheng $\mathrm{CH}$. Rhodium(III)-catalyzed synthesis of cinnolinium salts from azobenzenes and alkynes: application to the synthesis of indoles and cinnolines. Chem-Eur J 2013; 19 (20): 6198-202.

[6] Klenov MS, Ratnikov MO, Churakov AM, Solkan VN, Strelenko YA, Tartakovsky VA. Synthesis of [1,2,5]oxadiazolo[3,4-c]cinnoline 5-oxides. Russ Chem Bull 2011; 60 (3): 536-47.

[7] Zhang GW, Miao JM, Zhao Y, Ge HB. Copper-catalyzed aerobic dehydrogenative cyclization of N-methyl-N-phenylhydrazones: synthesis of cinnolines. Angew Chem Int Ed 2012; 51 (33): $8318-21$.

[8] Ball CJ, Gilmore J, Willis MC. Copper-catalyzed tandem C-N bond formation: an efficient annulative synthesis of functionalized cinnolines. Angew Chem Int Ed 2012; 51 (23): 5718-22. 
Scheme 1 Synthesis of compound $\mathbf{1}$.

Fig. 1 Crystal structure of the $1 /$ acetone complex with atom numbering. Thermal ellipsoids are plotted at 50\% probability level. Hydrogen atoms are omitted for clarity.

\section{Scheme 1}
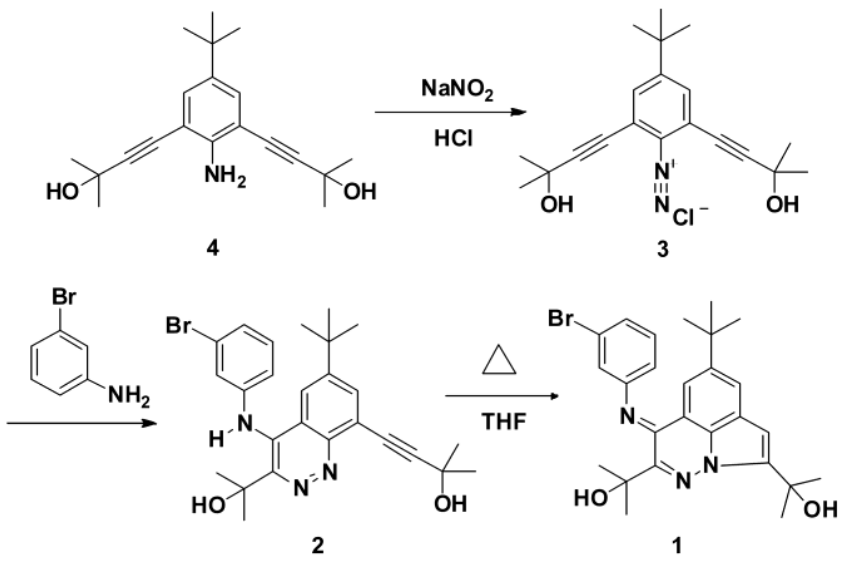

Fig. 1.

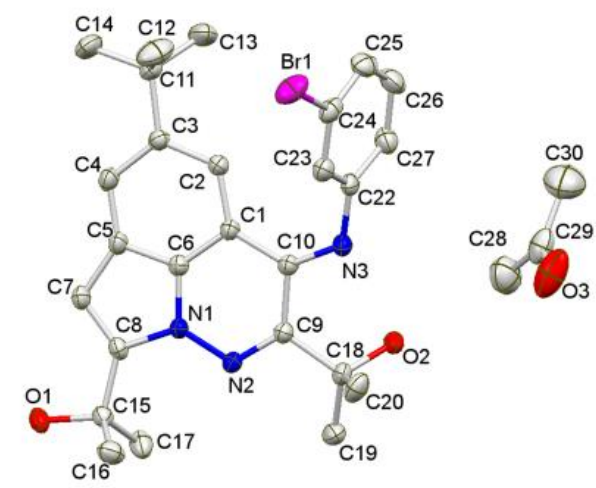




\title{
Synthesis, crystal structure and spectroscopic properties of a novel tricyclic cinnoline derivative
}

\author{
Yuehua Yuan ${ }^{a}$, Maozhong Tian ${ }^{\text {a,*, }}$ Qiangfeng Yin ${ }^{\text {b }}$ Feng Feng ${ }^{\text {a,* }}$ \\ ${ }^{a}$ College of Chemistry and Environmental Engineering, Shanxi Datong University, Datong \\ 037009, P. R. China. \\ ${ }^{\mathrm{b}}$ Product Engineering Department, Chemical Engineering Institute,
}

East China University of Science and Technology, Shanghai 200237, P. R. China.

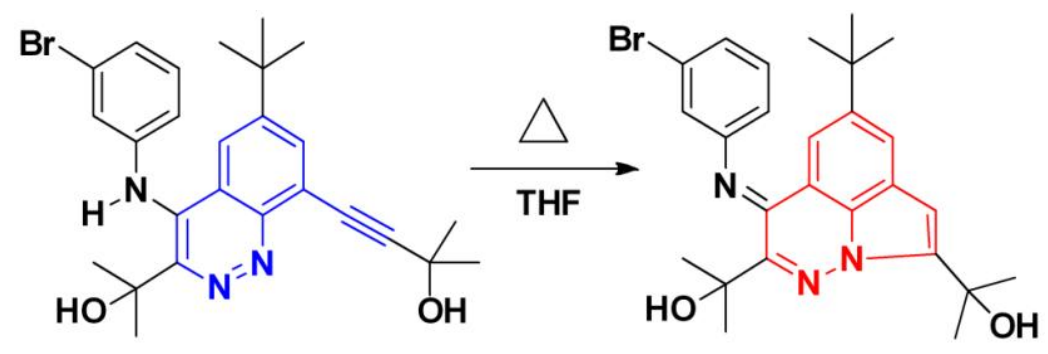

An unexpected tricyclic cinnoline derivative (E)-2,2'-(3-((3-bromophenyl) imino)-5-(tert-butyl)-3H-pyrrolo[3,2,1-ij]cinnoline-2,8-diyl)bis(prop-an-2-ol) was synthesized. The inclusion compound was formed between the tricyclic cinnoline and acetone in the crystal. 